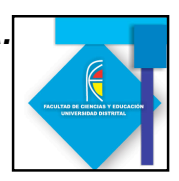

\title{
EL PRINCIPIO DE SUPERPOSICIÓN DE ESTADOS, A PARTIR DE LOS ESTADOS DE POLARIZACIÓN DE UNA ONDA MONOCROMÁTICA
}

\section{THE PRINCIPLE OF SUPERPOSITION OF STATES, FROM THE STATES OF POLARIZATION OF A MONOCHROMATIC WAVE}

\author{
Diana Milena Mendoza Cely ${ }^{1}$ \\ Mauricio Rozo Clavijo²
}

\section{Resumen}

En este trabajo se presenta una reflexión alrededor del principio de superposición de estados, partiendo del análisis del concepto de estado dentro del contexto de la mecánica clásica y el contexto de la mecánica cuántica. Además se pretende examinar de qué manera se implementa la idea de superposición, a partir del análisis de los estados de polarización de una onda monocromática. El principio de superposición de estados siendo uno de los principios fundamentales en la mecánica cuántica, tiene un grado de dificultad entre los estudiantes, ya que no es mediato entender que un sistema pueda estar en un estado como una combinación lineal de otros estados. De esta manera se utilizan analogías como herramienta didáctica dentro del contexto clásico y cuántico, con el fin de conceptualizar y establecer las diferencias del principio de superposición desde la teoría clásica y la teoría cuántica.

Palabras Clave: Estado, principio de superposición, Polarización, analogías, didáctica.

\begin{abstract}
:
A reflection on the principle of superposition of states is presented, from the analysis of the concept of state within the context of classical mechanics and quantum mechanics context. Also, it aims to examine how it implements the idea of superposition, based on the analysis of states of polarization of a monochromatic wave. The principle of superposition of states is one of the fundamental principles of quantum mechanics, has a degree of difficulty among students, it is not mediate understand that a system can be in a state as a linear combination of other states. In this way, analogies are used as a teaching tool within the context classical and quantum, to conceptualize and establish the principle of superposition differences from the classical theory and quantum theory.
\end{abstract}

Keywords: State, principle of superposition, polarization, analogies, didactic.

\footnotetext{
${ }^{1}$ Universidad Pedagógica Nacional, diana-8989@hotmail.com

${ }^{2}$ Universidad Pedagógica Nacional, mclavijo@pedagogica.edu.co
} 


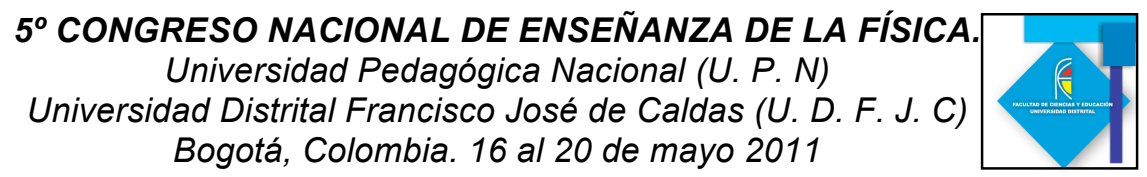

\section{Introducción:}

La indagación sobre el comportamiento de la materia a nivel atómico, condujo a los pensadores del siglo XIX a la elaboración de experimentos cuyos resultados pusieron en evidencia que tanto la mecánica newtoniana como la teoría clásica de campos, no daban respuesta satisfactoria de los datos empíricos obtenidos. Es así, que preguntas como: ¿qué es la Materia?, ¿tiene alguna estructura?, ¿cómo se explican sus propiedades?, no tienen respuesta dentro de las teorías clásicas de la física que sean concordantes con los datos experimentales.

El tratamiento dado a los fenómenos atómicos está relacionado con la idea de lo grande y lo pequeño del sistema a tratar, caracterizada por el hecho de que la observación que se realice sobre un sistema tiene un grado de perturbación incontrolable sobre éste . La nueva forma para la descripción de los fenómenos a nivel atómico, requiere de ideas que permitan lograr una imagen inteligible de los fenómenos a nivel atómico. Una de estas ideas es el principio de superposición de estados, el cual se puede establecer a partir de la polarización de una onda monocromática, como lo hace notar Dirac en su texto: Principios de Mecánica Cuántica.

El principio de superposición de estados tiene un grado de dificultad entre los estudiantes, ya que no es mediato comprender que un sistema pueda estar en un estado como una combinación lineal de otros estados. Las imágenes mentales que se hacen los estudiantes respecto a la linealidad, no se hacen evidentes en su aprendizaje. Para lograr una comprensión del principio de superposición de estados, se realiza un análisis en relación a la polarización de una onda monocromática, para examinar en qué contexto se implementa la idea de superposición.

Por otro lado las investigaciones que se han realizado sobre la enseñanza de la mecánica cuántica, parten de los conceptos fundamentales de la teoría, como el principio de superposición y el principio de correspondencia. Es así como Fanaro (2009) propone centrar la atención en técnicas que pueden servir para la enseñanza. Una de ellas es la de caminos múltiples utilizada por Feynman (1970), en la cual Fanaro propone un diseño didáctico para analizar el comportamiento cuántico de los electrones a través de la experiencia de la doble rendija.

\section{El principio de superposición desde el contexto de la mecánica clásica}

La idea de estado juega un papel importante para describir la evolución temporal de un sistema clásico. Desde este contexto, se define el estado de un sistema como las diferentes formas de estar, sin que el sistema pierda su identidad. Además, el estado está relacionado con la idea de cualidad; cualidad que puede ser térmica, eléctrica, etc. De esta manera, se puede afirmar que al hablar del estado térmico de un sistema, se está refiriendo a la cualidad térmica en la que se puede encontrar, es decir; caliente, frio, tibio, etc. 


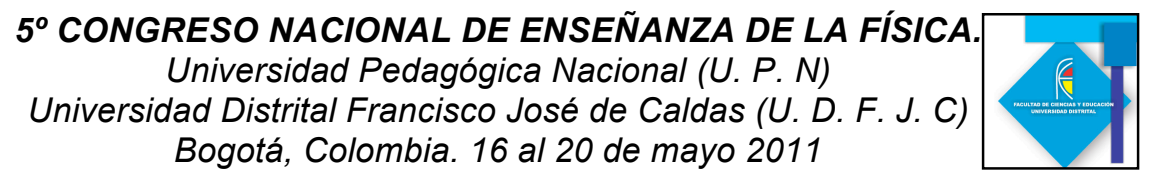

El estado de un sistema clásico, se determina en relación a las variables dinámicas de posición y momentum $x(t) ; P(t)$. Teniendo en cuenta que dichas variables se pueden medir simultáneamente, sin que el sistema se perturbe al realizar la observación sobre este. Por ejemplo, al describir el estado de un objeto que dejamos caer a una determinada altura, se puede medir la posición y el momentum del sistema en cualquier intervalo de tiempo determinado sin que el sistema sea perturbado por la observación.

Teniendo en cuenta la descripción realizada en torno al estado de un sistema, podemos dar paso hacia la definición del principio de superposición. El Principio de superposición está definido en relación a la operación aditiva de estados que hacen parte de la combinación lineal. En esta combinación lineal de estados, el sistema no pierde su identidad, es decir, los estados no se mezclan. Por ejemplo, al preparar un café con leche, mezclamos el café y la leche, sin embargo al terminar la preparación no podemos separar el café de la leche, es decir, al mezclar se pierde la identidad de los elementos que entran en la combinación.

Al referirnos al principio de superposición de estados, se puede tomar como analogía la superposición de movimientos como se muestra en la Figura 1. Cuando se combina un movimiento horizontal con velocidad constante y un movimiento vertical con aceleración constante, generan un movimiento que describe una trayectoria parabólica. Cada uno de los movimientos de la combinación no pierden su identidad, sin embargo combinados generan una persección diferente. Este es un ejemplo donde la superposición de dos movimientos, movimiento parabólico, se percibe diferente a cada uno de los movimientos componentes, el horizontal y el vertical, sin que ninguno de éstos pierda su identidad.

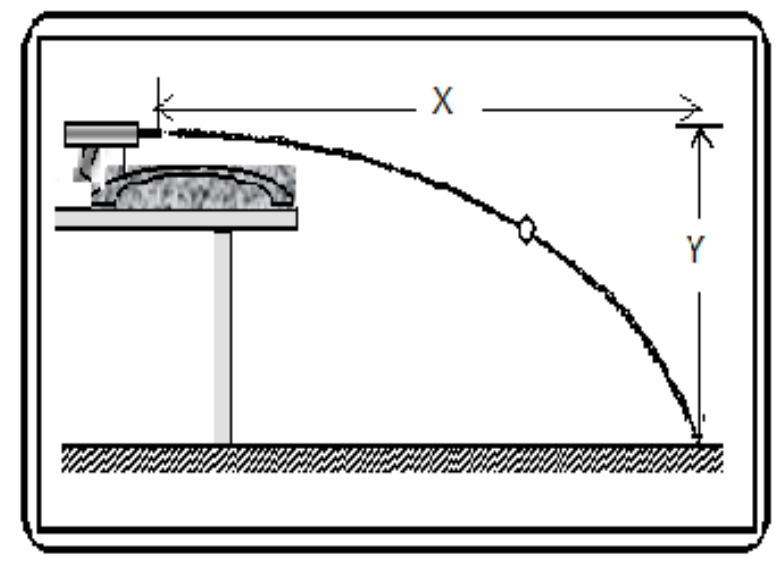

Figura 1. Superposición de movimientos

Como otro ejemplo se puede considerar, la superposición de magnitudes escalares. Al sumar dos números nos da como resultado un nuevo valor, donde los números componentes no pierden su identidad, es decir, no son una mezcla de números, sino simplemente la superposición de dos magnitudes, como se muestra en la ecuación 1,

$$
|11>=\beta| 5>+\alpha \mid 6>\quad \beta=1 \quad y \quad \alpha=1 . \quad \text { Ecuación } 1
$$


En los ejemplos anteriores, se resalta la idea de estado como una forma de estar del sistema, relacionado con la idea de cualidad. El estado de un sistema clásico se determina en relación a las variables dinámicas, donde el sistema no se perturba al realizar la medición éstas. Además, en la superposición de estados cada uno de sus constituyentes no pierden su identidad, simplemente generan una percepción diferente al ser combinados.

Con el surgimiento de la mecánica cuántica, se puso en tela de juicio la capacidad que tiene el hombre de conocer. Para conocer un objeto es necesario que interactuemos con él. Interacción que desde el contexto de la mecánica cuántica, implica que el objeto sea perturbado por el observador al realizar una observación sobre este. Desde este contexto, el sujeto se encuentra limitado respecto a la información que recibe del objeto, la cual puede ser alterada por el sujeto al realizar la interacción (G.Bautista. Sobre el conocimiento).

\section{El principio de superposición de estados desde el contexto de la mecánica cuántica.}

En mecánica cuántica existen limitaciones respecto a la precisión en la medición de magnitudes dinámicas sobre los sistemas a nivel atómico. Dirac, en su texto "Principios de mecánica Cuántica" introduce una discusión alrededor de lo pequeño y grande. Cuando un objeto no es perturbado por el observador al realizar una medición sobre éste, se dice que el objeto es grande, sin embargo, si el objeto es perturbado por la observación será considerado pequeño.

La idea de lo grande y pequeño toma un papel fundamental en física, ya que todo objeto considerado como grande estará sometido al principio de causalidad y su movimiento quedará descrito por ecuaciones diferenciales dependientes del tiempo. Por otro lado, si el objeto es considerado pequeño en relación con la perturbación producida, implica establecer si el principio de causalidad sigue siendo válido. Manteniendo la validez de este principio, conduce a realizar una descripción del estado del sistema en términos probabilísticos, es decir, la máxima información que se puede obtener sobre el estado de uno de los estados componentes de la superposición, es la probabilidad de que el sistema se encuentre en este estado.

La idea de linealidad juega un papel importante para la descripción de los fenómenos a nivel atómico, caracterizada por la superposición de estados sin que estos pierdan su identidad. Cuando se considera un haz de luz constituido por fotones, cada uno de ellos polarizado según una dirección determinada, se puede describir el estado del sistema a partir de la superposición de los estados componentes. Cuando el haz de luz esta polarizado oblicuamente, el fotón puede ser descrito como la superposición de dos estados, paralelo y perpendicularmente, sin que se pierda la identidad de cada uno, como se muestra en la ecuación 2 ,

$$
\mid \text { El estado del foton }\rangle=\beta \mid \text { paralelo }\rangle+\alpha \mid \text { Perpendicularmente }\rangle \quad \text { Ecuacion } 2 .
$$




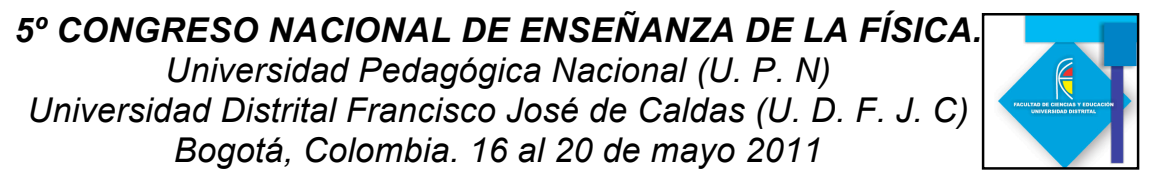

En este ejemplo, se propone el caso de un fotón polarizado oblicuamente, donde el estado del fotón puede ser descrito como la superposición de dos estados: uno paralelo y uno perpendicular. De esta manera se considera que el estado de polarización del fotón, puede ser expresado como una superposición de dos estados.

\section{Referencias Bibliográficas}

Antonio, E. Gallarreta, S. (2006). Aportes para la utilización de analogías de la enseñanza en la enseñanza de las ciencias. Ejemplos en biología del desarrollo. Revista Iberoamericana de educación. 1-9.

Dirac, P. (1976) El Principio de la Mecánica Cuántica. Fourth edición. I capitulo. El principio de superposición (pp.15-34).

Fanaro, M. (2009). La Enseñanza de la Mecánica Cuántica en la escuela Media. Tesis Doctoral. Burgos 2009. Memoria para optar al título de Doctor, Universidad de Burgos.

González, B. (2005). El modelo analógico como recurso didáctico en ciencias experimentales. Revista Iberoamericana de educación.1-15.

Moreno, H. Guarín, E. (2010). Nociones cuánticas en la escuela secundaria: Un estudio de caso. Latin American Journal of Physics Education. (4). (3). 669-676.

Organista, O. Gómez, D. Rodríguez, J.(2007). Una idea profunda en la comprensión del mundo físico: el principio de superposición de estados. Latin American Journal of Physics Education. (1). (1). 83-88.

Otero, M. Fanaro, M. Arlego, M. (2009). Investigación y desarrollo de propuestas didácticas para la enseñanza de la física en la escuela secundaria: Nociones cuánticas. Revista electrónica de investigación en ciencias.vol 4. 58-74.

Peña, M.(2008) Un Acercamiento Al Concepto De Estado En la Teoría Cuántica. Monografía de la universidad Pedagógica Nacional. Trabajo de grado para optar al título de licenciado en física. 